\title{
Fabrication of a Resonance Absorption-Type Microstrip Line Isolator Using Polycrystalline YIG
}

\author{
M. KIM, D. S. JUN, J. R. PARK. S. S. LEE
}

Component Technology Development Section, Electronics and Telecommunications Research Institute, 161 Kajung-Dong, Yusung-Gu, Taejon, 305-350, Korea

\begin{abstract}
In response to rapidly increasing demands on mobile and satellite communication services, microwave devices using ferrite materials have received much attention from scientific communities around the world. This paper presents the design and fabrication of the microstrip isolator operating at the frequency band of $2.15 \mathrm{GHz}$. The isolator was designed to reduce the size by the magnetic wall ridges. The commercial software using a 3D finite element method was used to analyze the scattering matrix of the microstrip isolator. Polycrystaline garnet was used for ferrite substrate. The isolator shows insertion loss $0.2 \mathrm{~dB}$, return loss $30 \mathrm{~dB}$, isolation $30 \mathrm{~dB}$ at $2.15 \mathrm{GHz}$.
\end{abstract}

\section{INTRODUCTION}

The design procedure for classical stripline isolators has been known since the mid 1960's. Fay and Comstock[1] proposed $\mathrm{Y}$-junction to be considered as a nonreciprocal resonator operated with two counter-rotating modes. The theory of the junction circulator using triangular resonators has been treated with the assumption for magnetic walls and by neglecting of the fringing fields by a number of authors[2],[3]. Planar triangular resonators with magnetic side wall boundary conditions symmetrically loaded by rectangular magnetic wall ridges were investigated to reduce the size and to tune the frequency.

This paper presents a microstrip isolator with nonsymmetric magnetic wall ridges as a tuning means. The 3-D finite element software adopted in this work is Ansoft HFSS5.0. It can deal with 3D geometry and consider the fringing field, so that the original circuit was analyzed with high accuracy.

\section{ELECTRIC FIELD PATTERNS OF TRIANGULAR RESONATORS}

The four structures dealt with in this work are shown in Fig. 1. In this paper, the electric field patterns and the fundamental-mode cutoff wavenumber of the four resonators and isolator have been investigated using the commercial 3D finite element program package.

$$
\begin{aligned}
& \left(\nabla_{x, y, z}^{2}+k^{2} \text { eff }\right) E_{x, y, z}=0 \\
& k_{e f f}^{2}=\omega^{2} \varepsilon_{o} \varepsilon_{e f f} \mu_{o} \mu_{e f f} \\
& \mu_{e f f}=\frac{\mu^{2}-\kappa^{2}}{\mu}
\end{aligned}
$$

$$
\text { where }\|\mu\|=\left|\begin{array}{ccc}
\mu & -j \kappa & 0 \\
j \kappa & \mu & 0 \\
0 & 0 & 1
\end{array}\right|
$$

For the pure triangular configurations with unmagnetized ferrite $\mu=1$, the cutoff number for the dominant mode in the absence of magnetic ridges is given by [4]

$$
k A=4 * \pi / 3
$$

In the above equations $\mathrm{A}$ is the length of side of the planar triangular resonator in meters, and $\mathrm{k}$ is the radial propagation constant.

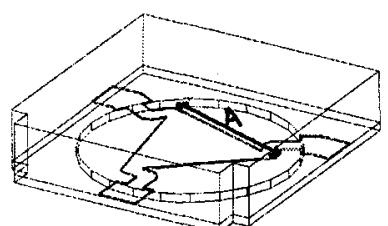

(a) pure triangular resonator

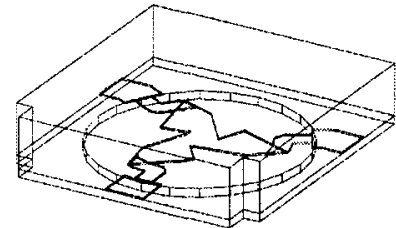

(c) with deep triangular ridges

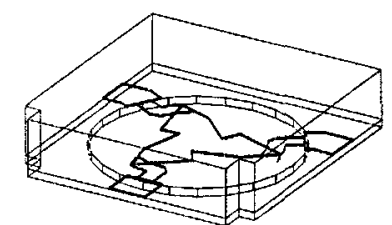

(b) with small triangular ridges

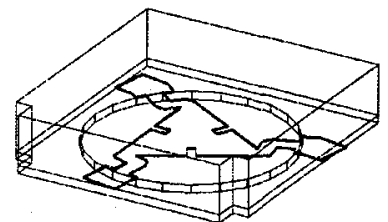

(d) with rectangular ridges
Fig. 1.Triangular Resonators with several magnetic wall ridges for isolators. (The side length $A$ of the triangular resonators are same)

With the four circuits in Fig. 1, the thickness of the ferrite substrate, the thickness of the metal, the width of ports, the width of transformers, dielectric constant were $1 \mathrm{~mm}, 0.2 \mathrm{~mm}, 4.57 \mathrm{~mm}, 2.07 \mathrm{~mm}, 14$, respectively. The frequency response and electric field pattern of the ferrite resonators which are not magnetized are calculated by the software with 6000 tetrahedral elements. The effect of magnetic wall ridges in triangular resonators on the variation of the resonant frequencies is shown in Fig. 2. For the configuration in Fig. 2(a), the cutoff number for the dominant mode is given by $\mathrm{kA}=3.15$. For the configuration in Fig. 2 (b) (d), the cutoff number of the dominant mode is given by $\mathrm{kA}=2.8, \mathrm{kA}=2.6, \mathrm{kA}=2.96$, respectively. As increasing the size of the triangular ridges, the resonant frequency of the resonators is reduced in Fig. 2 (a) (c). Triangular ridges show more efficient frequency shift than rectangular ridges do in Fig. 2 (b), (d). 

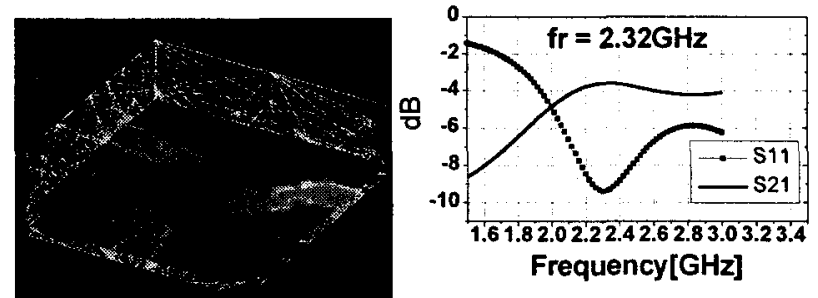

(a) Triangular resonator without magnetic wall ridges.
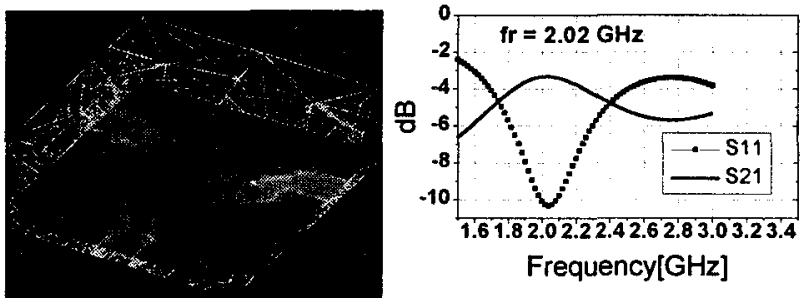

(b) Triangular resonator with triangular ridges.
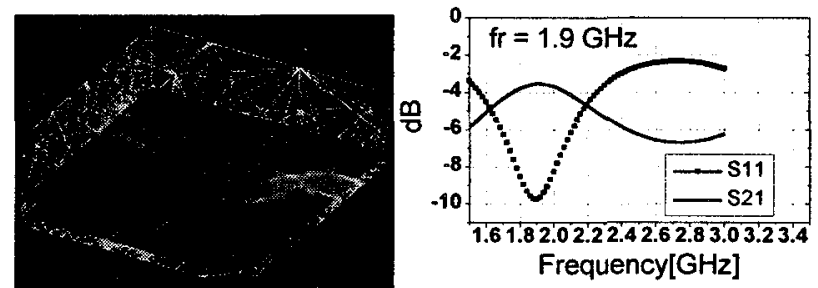

(c) Triangular resonator with deep triangular ridges.
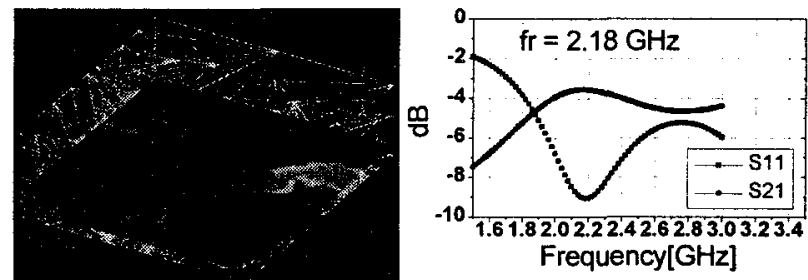

(d) Triangular resonator with square ridges.

Fig. 2. Field patterns and frequency responses of the triangular resonators with different ridges

III. ISOLATORS USING TRIANGULAR RESONATORS WITH MAGNETIC WALL RIDGES

The isolator operating at $2 \mathrm{GHz}$ is consisted of a ground conductor, ferrite materials, $50 \Omega$ load, and hard magnet in Fig. 3. The radius of the circular ferrite was $12.5 \mathrm{~mm}$, the radius of the hard magnet was $5 \mathrm{~mm}$. The $\mathrm{DC} \mathrm{H}$ field strength by the magnet is $500 \mathrm{Oe}$.

Polycrystalline $\mathrm{Y}_{2.4} \mathrm{Ca}_{0.3} \mathrm{Sn}_{0.3} \mathrm{Fe}_{3.8} \mathrm{Al}_{1.2} \mathrm{O}_{12}$ garnet was chosen to verify the results of FEM analysis. The specifications of the garnet are saturation magnetization $680 \mathrm{G}$, curie temperature $285^{\circ} \mathrm{C}$, temperature coefficient of saturation magnetization $0.2 \% /{ }^{\circ} \mathrm{C}$, ferrimagnetic resonance line width $(\Delta \mathrm{H}) 40 \mathrm{Oe}$, dielectric constant $\varepsilon_{r} 14$, dielectric loss $\tan \delta \quad 0.0004$. The magnetization, ferrimagnetic resonance line-width of the ferrite material and the applied magnetic field are main parameters to design isolators.

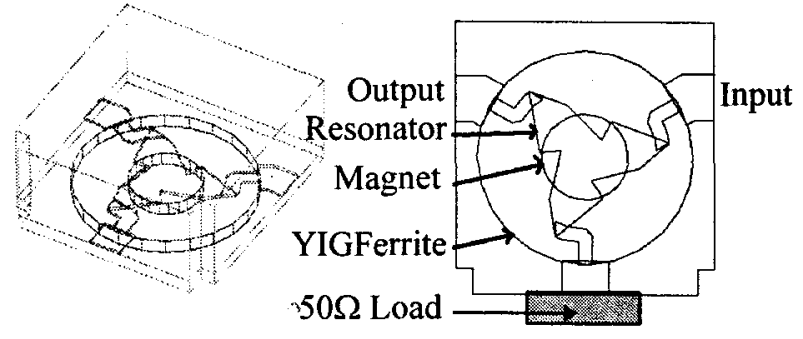

(a) Structure of the isolator.

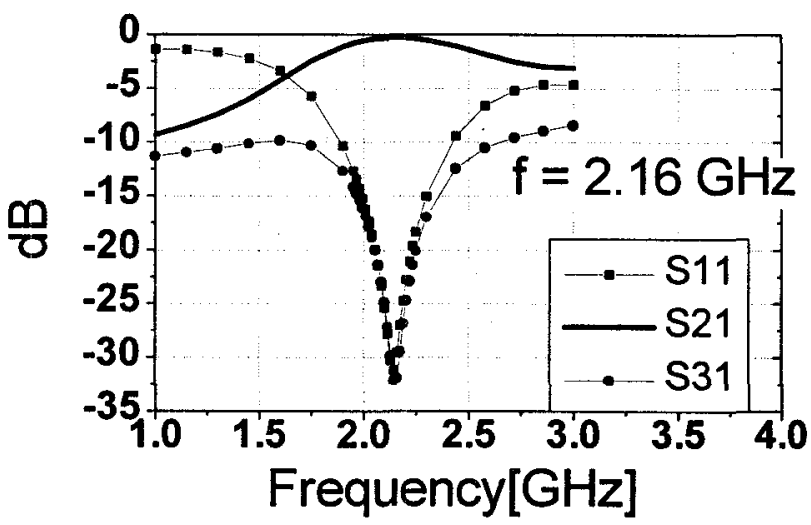

(b) Frequency response of the isolator.

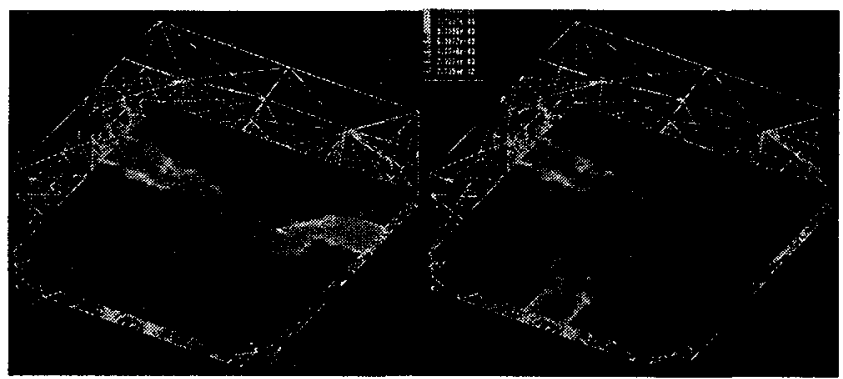

(c) E-fields pattern at the center frequency.

Fig. 3. Simulated results of the microstrip isolator using triangular resonator.

The isolator presented in this work operates at the below resonance condition. In the case of the triangular resonator with small triangular ridges, the A dimension was held constant at $17.3 \mathrm{~mm}$ and thickness of the ferrite was $1 \mathrm{~mm}$. The triangular magnetic wall ridge was held constant at $5.5 \mathrm{~mm}, 5.76 \mathrm{~mm}$, and $2.45 \mathrm{~mm}$. This isolator was implemented by attaching $50 \Omega$ load on the one port of circulator rotating to the counterclockwise direction. The internal DC magnetic field of the ferrite substrate is 0 Oe. The isolator shows Insertion loss $0.28 \mathrm{~dB}$, Return loss $30 \mathrm{~dB}$, Isolation $32 \mathrm{~dB}$, with $\mathrm{k} / \mathrm{u}=0.88$ at $2.16 \mathrm{GHz}$. The frequency response of the isolator is calculated by $3 \mathrm{D}$ FEM. The magnitude of the E field of the isolator are shown at the top plane of YIG ferrite in Fig. 3. 


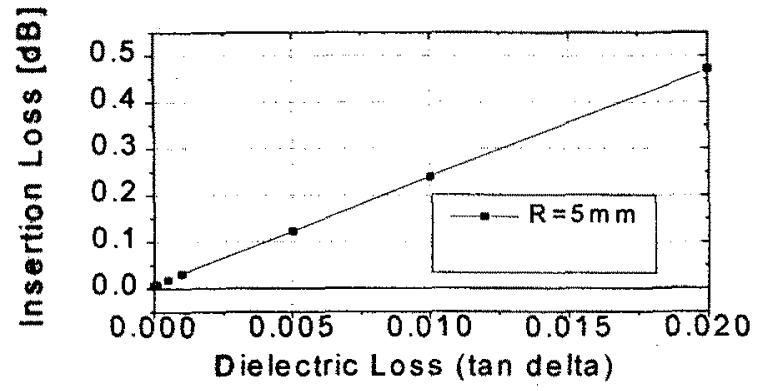

Fig. 4. Effects of dielectric loss $(\tan \delta)$ on the insertion loss of the isolator

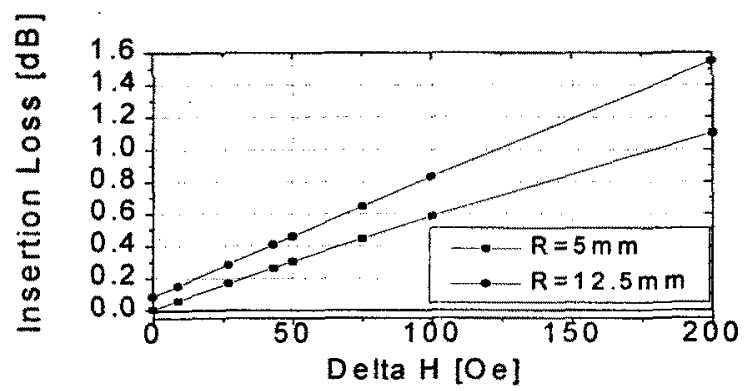

Fig. 5. Effects of magnetic loss $(\Delta H)$ on the insertion loss of the isolator.

The insertion loss of the isolator has been investigated by varying the dielectric loss $\tan \delta$ and ferrimagnetic resonance linewidth $(\Delta H)$. As increasing dielectric loss and magnetic loss terms, the insertion loss increases linearly in Fig. 4,5. As increasing the size of the magnet, the insertion loss increases due to the increase of the magnetic loss area in Fig. 5 .

Therefore the isolator with low insertion loss and small size can be incorporated by reducing the size of the magnet and the loss terms of the ferrite material.

In Fig. 6, the experimentally measured characteristics of the isolator with a center frequency $2.15 \mathrm{GHz}$ are presented. It shows Insertion loss $0.2 \mathrm{~dB}$, Return loss $30 \mathrm{~dB}$, Isolation $30 \mathrm{~dB}$ at $2.15 \mathrm{GHz}$.

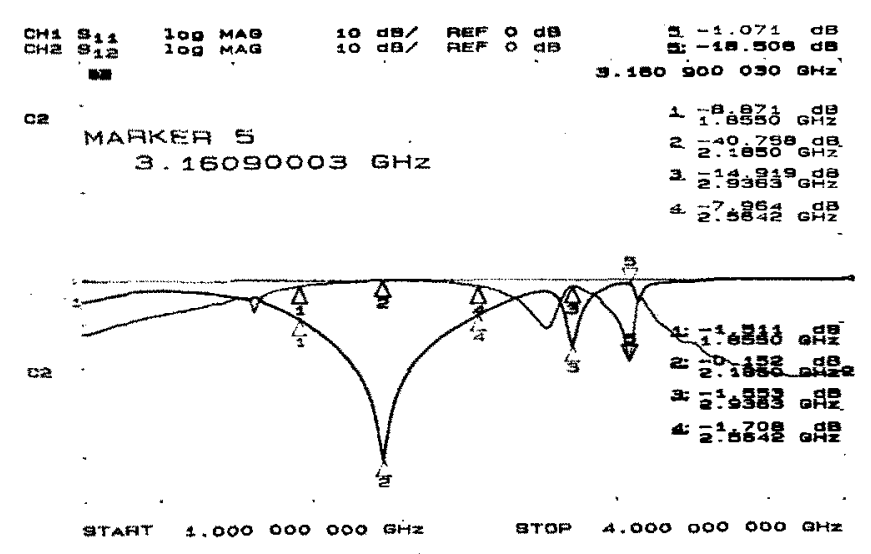

(a)Insetion loss and return loss.

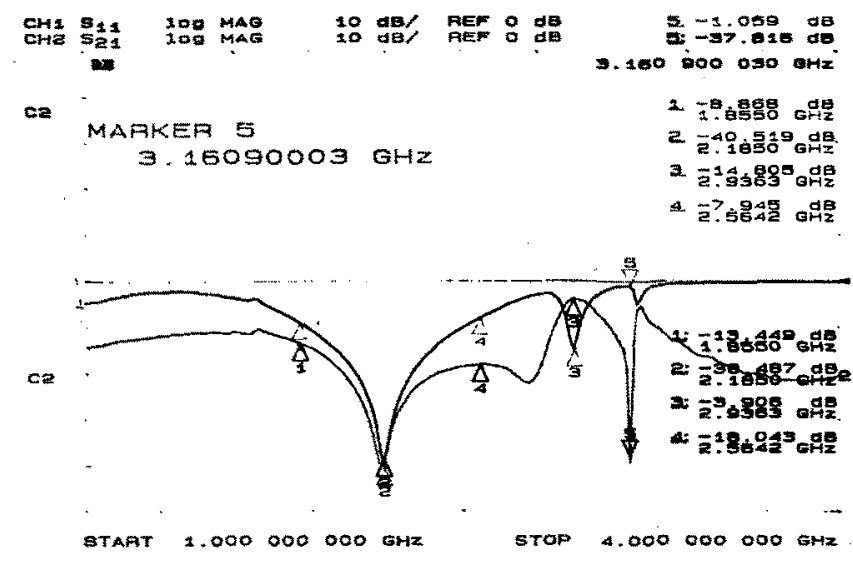

(b) Isolation.

Fig. 6. Experimental performance of the isolator with magnetic wall ridges.

In the experiment, however, the number of the meshes and the difference between uniform dc magnetic field assumed in FEM simulator and experimental magnetic field cause a little different results in Fig. 5, 6. A comparison of the experimental and simulated results shows good agreements. The isolator was designed to have good characteristics at the narrow band. As a result of the nonsymmetric triangular ridges, the resonator of the isolator is nonsymmetric type.

\section{CONCLUSIONS}

This paper has presented a simulated and experimental investigation of triangular resonators with triangular magnetic wall ridges to reduce the size of the resonator. The simulations of the resonators and isolator have been calculated using a finite element program software. Good agreement between simulation and experiment was obtained.

\section{REFERENCES}

[1] C. E. Fay, and R. L. Comstock, "Operation of the Ferrite Junction Circulator," IEEE Trans. Microwave Theory Tech., vol. MTT-13, pp. 15-27, Jan. 1965.

[2] J. Helszajn, D. S. James, and W. T. Nisbet, "Circulators Using Planar Triangular Resonators," IEEE Trans. Microwave Theory Tech., vol. MTT-27, pp. 188-193, Feb. 1979.

[3] R. W. Lyon, and J. Helszajn, "A Finite Element Analysis of Planar Circulators Using Arbitrarily Shaped Resonators," IEEE Trans. Microwave Theory Tech., vol. MTT-30, pp. 1964-1974, Nov. 1982.

[4] J. Helszajn, R. D. Baars, and W. T. Nisbet, "Characteristics of Circulators Using Planar Triangular and Disk Resonators Symmetrically Loaded with Magnetic Ridges," IEEE Trans. Microwave Theory Tech., vol. MTT-28, pp. 616-621, June. 1980. 